\title{
Spin dynamics calculations of electron and nuclear spin relaxation times in paramagnetic solutions
}

\author{
Shawn M. Abernathy and Robert R. Sharp \\ Department of Chemistry, The University of Michigan, Ann Arbor, Michigan 48104
}

(Received 16 December 1996; accepted 4 March 1997)

\begin{abstract}
Spin dynamics (SD) methods have been developed to compute NMR paramagnetic relaxation enhancements (NMR-PRE) produced by solutes with electron spin $S \geqslant 1$ in solution. The spin dynamics algorithms, which are implemented as the computer program SpinDyn.f, are similar in spirit to molecular dynamics calculations in statistical mechanics, except that the spin motion is propagated numerically in time using quantum mechanical equations of motion of the spin operators, rather than Newtonian equations of motion of the molecular degrees of freedom as in MD simulations. SD simulations as implemented in SpinDyn.f provide accurate, flexible, and rapid calculations of NMR-PRE phenomena with few of the assumptions or limitations of previous analytical theories. The program calculates inter- and intramolecular NMR-PRE phenomena for both integer and half-integer spin systems processing under arbitrary Zeeman and zfs Hamiltonians in the presence of Brownian reorientation. Isotropic Brownian reorientation is simulated by means of a finite-step algorithm with adjustable step size. Simulations computed by SpinDyn.f have been used in a systematic study aimed at better understanding the influence of Brownian reorientation on the NMR-PRE of an $S=1$ ion in a non-Zeeman-limit physical situation. Conditions required for the validity of zfs-limit analytical theory are given. SpinDyn.f has also been used to assess quantitatively the effects of molecular reorientation on a prior analysis of NMR-PRE data for the model $S=2$ complex ion [tris-(acetylacetonato)manganese(III)] in acetone solution; this system was found to be well described by zfs-limit analytical theory. (C) 1997 American Institute of Physics.
\end{abstract} [S0021-9606(97)01022-2]

\section{INTRODUCTION}

NMR paramagnetic relaxation enhancements (NMRPRE), i.e., the enhancements of NMR relaxation rates that are produced by paramagnetic species in solution, have been used widely as a source of experimental information concerning molecular structure, Brownian motion, and chemical exchange kinetics in solution. They have also provided valuable information concerning the electron spin relaxation properties of paramagnetic ions. For electron spins $S \geqslant 1$, the zero-field splitting (zfs) interaction is nonzero (except by reason of symmetry), and the theoretical analysis of NMR-PRE phenomena is complicated by the nature of the spin precessional motion which occurs in the presence of competing Zeeman and zfs interactions. At high laboratory magnetic field strengths where the Zeeman Hamiltonian is much larger than the zfs Hamiltonian $\left(H_{\text {Zeem }} \gg H_{\text {ZFS }}\right)$, the precession of the electron spin is quantized along the external laboratory magnetic field $\bar{B}_{o}$. This situation is described by the traditional Zeeman-limit theory developed by Solomon, ${ }^{1}$ Bloembergen, ${ }^{2,3}$ and Morgan ${ }^{3}$ (SBM theory). At low magnetic field strengths $\left(H_{\text {Zeem }} \ll H_{\text {ZFS }}\right)$, the precession of the electron spin is quantized along molecule fixed axes, specifically along the principal axis system of the zfs tensor (zfsPAS). Analytical zfs-limit expressions, which parallel in form those of SBM theory, have been derived for both intramolecular ${ }^{4}$ and intermolecular ${ }^{5}$ electron-nuclear relaxation. In the intermediate regime where $H_{\text {Zeem }} \approx H_{\text {ZFS }}$, the precessional motion becomes more complex as the quantization axes shift between the external field direction and the
zfs-PAS. Analytical theory has also been developed to describe NMR relaxation in this intermediate situation, ${ }^{6-14}$ but only in the limit of slow molecular reorientation, in which the motion of the electron spin is treated as it would be in a glass. This approach, for which computer implementations are available, ${ }^{10,14}$ is appropriate when electron spin relaxation is rapid compared to molecular reorientation. This is expected to be the case of macromolecules but not necessarily for low molecular weight solutes.

Fast molecular reorientation of a paramagnetic solute introduces stochastic time dependence into the electronnuclear dipole-dipole coupling interaction, which can have important effects on NMR-PRE phenomena, and which appear in the theory in three ways. First, in the intermediate regime $\left(H_{\text {Zeem }} \approx H_{\mathrm{ZFS}}\right)$, the electron spin precessional frequencies depend on the relative orientation of the molecular and laboratory coordinate frames, so that Brownian reorientation introduces a stochastic time dependence into the precessional motion. Secondly, stochastic time dependence in the zfs interaction provides a physical mechanism of electron spin relaxation. And thirdly, reorientation of the quantization axes of the electron spin modulates the spatial variables of the $I-S$ dipolar coupling. For these reasons, fast molecular reorientation introduces strong coupling between the motions of the spin and spatial variables, so that their ensemble averages cannot be computed separately.

The closed-form Zeeman-limit (SBM) and uniaxial zfs-limit ${ }^{4,5}$ expressions do account for effects of reorientational motions by including appropriate terms in the dipole- 
dipole correlation time (see below), but the effects of reorientational motions have not been incorporated in the analytical theories that describe the intermediate regime $\left(H_{\text {Zeem }} \approx H_{\mathrm{ZFS}}\right)$; analytical theories appropriate to these latter situations can thus be called "slow motion" theories. Formalisms based on the stochastic Liouville equation (SLE) have been developed as a more general approach to the problem. For this purpose, the SLE has been formulated in a mixed basis of infinite dimension consisting of product functions of classical (spatial) and quantum mechanical (spin) variables, and solutions have been derived in two forms: as a hierarchy of coupled differential equations by Freed et al., ${ }^{15,16}$ and by Hwang and co-workers; ${ }^{17-19}$ and as the inverse of an approximate Liouvillian matrix, by Westlund, Kowalewski, and their co-workers. ${ }^{20-25}$ These formulations, which are complex, can be simplified considerably by the slow motion or "decomposition" approximation (i.e., independent averaging of spin and spatial variables), and this approximation has been used in the majority (but not all ${ }^{24}$ ) of practical applications (Ref. 26 is an excellent recent review of Swedish work).

This paper describes new methods for performing spin dynamics (SD) simulations of NMR-PRE phenomena in which the motion of the electron spin is calculated explicitly in the time domain from the quantum mechanical equation of motion using a time-dependent spin Hamiltonian to simulate the effects of molecular reorientation. The approach is analogous to that of molecular dynamics simulations of liquids, except that the time dependence of the spin operators is solved using quantum mechanical equations of motion rather than, as in molecular dynamics simulations, from Newtonian equations of motion of molecular degrees of freedom. We show below that the spin dynamics method, as implemented in the program SpinDyn.f, provides a flexible, accurate, and reasonably fast means of calculating nuclear and electron spin relaxation times under rather general physical conditions: the method simulates effects of Brownian reorientation in the presence of arbitrary Zeeman and zfs interactions of the electron spin. Among the advantages of SD simulations is the fact that they are not subject to the limitations of the Redfield theory, and thus provide a powerful platform for simulating electron spin precessional and relaxation behavior under quite general physical conditions. The program incorporates a finite-step algorithm with adjustable step size to simulate isotropic reorientation. The time-domain SD computational approach is very flexible and can readily be modified to accommodate more complex kinds of motion, as, for example, anisotropic reorientation, effects of internal motions, or models that incorporate both large- and small-step angular motions. ${ }^{27,28}$ This approach can also be combined with molecular dynamics simulations, thereby providing a much more realistic description of molecular motions than is possible using analytical mathematical models. The combined use of spin- and molecular dynamics simulations has been pioneered in recent work of Odelius et al. ${ }^{29,30}$ in a study of the hexaaquanickel (II) ion.

In work described below, we have used spin dynamics simulations as an aid in understanding the limits of applica- bility of the zfs- and Zeeman-limit analytical theories and for characterizing, qualitatively and quantitatively, the deviations from analytical theory that are produced by rapid Brownian reorientation. To carry out this objective, we have first tested the performance of the algorithms of SpinDyn.f against the results of prior analytical theory in the limiting slow motion regimes. Next, we have surveyed the nature and magnitude of departures from the limiting slow motion behavior that are produced by fast Brownian reorientation for an $S=1$ spin system, and explored the situations in which these effects can appropriately be described by zfs-limit theory. The simulations described below involve specifically the intermolecular (solvent-solute) NMR-PRE, although the theory developed describes both inter- and intramolecular relaxation, and the program SpinDyn.f handles both situations. The effects of Brownian reorientation on the intramolecular NMR-PRE are more complex than are reorientational effects in intermolecular relaxation, and we will address them in a subsequent communication.

Finally, we have reexamined, using SD simulations, a prior analysis ${ }^{5,31}$ of solvent ${ }^{1} H$ NMR-PRE data for the model $S=2$ system, [tris-(acetylacetonato)manganese(III)], which was analyzed previously using zfs-limit analytical theory. We used spin dynamics simulations to assess the accuracy of the simple zfs-limit analytical theory expressions in describing the NMR-PRE for a low molecular weight integer spin complex of this kind.

\section{THEORY}

We assume that nuclear spin relaxation is produced by magnetic dipole coupling between the nuclear spin $\bar{I}$ and an electron spin $\bar{S}$ (with spin quantum numbers $I, S$ ), for which the coupling Hamiltonian, written in spherical tensor form (SI units), is

$$
H_{d-d}=\frac{\kappa}{r^{3}} \sum_{q=-1}^{+1} 3^{-1 / 2}(-1)^{1-q} I_{q}^{(1)} F_{-q}^{(1)},
$$

with

$$
\begin{aligned}
& \kappa=-30^{1 / 2} \gamma_{I} g \beta_{0}\left(\frac{\mu_{0}}{4 \pi}\right), \\
& I_{ \pm 1}^{(1)}=\mp 2^{-1 / 2} I_{ \pm}, \\
& I_{0}^{(1)}=I_{z} .
\end{aligned}
$$

$\gamma_{I}$ is the nuclear spin magnetogyric ratio, $g$ is the electron spin $g$-value, $\beta_{0}$ is the Bohr magneton, $\mu_{0}$ is the magnetic permeability of free space, and $r$ is the $I-S$ interspin distance. $F^{(1)}$ is the first rank tensor formed from the "lattice" variables, $S_{x, y, z}$ and $(\theta, \varphi)$, the polar angles which specify the orientation of the interspin vector in the laboratory coordinate frame, where external magnetic field $\bar{B}_{0}$ defines the laboratory $z$ axis. $F^{(1)}$ is the first rank tensor product

$$
F^{(1)}=\left\{S^{(1)} \otimes C^{(2)}\right\}^{(1)},
$$

with components 


$$
F_{m}^{(1)}=\sum_{p=-1}^{1} \sum_{q=-2}^{2}(-1)^{1-q} 3^{1 / 2}\left(\begin{array}{c}
121 \\
p q m
\end{array}\right) S_{p}^{(1)} C_{q}^{(2)}(\theta, \varphi) .
$$

$S^{(1)}$ is the first rank tensor composed of the electron spin variables, the components $\left(S_{p}^{(1)}\right)$ of which are defined analogously to $I_{p}^{(1)} \cdot C_{q}^{(2)}(\theta, \varphi)$ are components of the second rank tensor formed by the polar variables $(\theta, \varphi)$, given by

$$
C_{q}^{(2)}(\theta, \varphi)=(4 \pi / 5)^{1 / 2} Y_{q}^{2}(\theta, \varphi)
$$

where $Y_{q}^{2}(\theta, \varphi)$ are spherical harmonics.

Evaluation of the 3-j symbols in Eq. (6) gives the following explicit form for the components of $F^{(1)}$,

$$
F_{m}^{(1)}=\left(\frac{4 \pi}{5}\right)^{1 / 2} \sum_{p=-1}^{1} c_{m, p} S_{p}^{(1)} Y_{m-p}^{(2)}(\theta, \varphi)
$$

with

$$
\begin{aligned}
& c_{+1,+1}=c_{-1,-1}=10^{-1 / 2}, \\
& c_{+1,0}=c_{-1,0}=-3^{1 / 2} \cdot 10^{-1 / 2}, \\
& c_{0,+1}=c_{0,-1}=3^{1 / 2} \cdot 10^{-1 / 2}, \\
& c_{+1,-1}=c_{-1,+1}=3^{1 / 2} \cdot 5^{-1 / 2}, \\
& c_{0,0}=-2^{1 / 2} \cdot 5^{-1 / 2} .
\end{aligned}
$$

The dipole-dipole part of the paramagnetic relaxation enhancement for the nuclear spin $T_{1}$ and $T_{2}$ relaxation rates can be written in terms of the components of $F^{(1)}$ as

$$
\begin{aligned}
\left(R_{1 p, d}\right)^{-1}= & 3^{-1} \kappa^{2} \int_{0}^{\infty}\left\{\left\langle\frac{F_{+1}^{(1)}(t)}{r^{3}} \frac{F_{-1}^{(1)}(0)}{r_{0}^{3}}\right\rangle \exp (-i \omega t)\right. \\
& \left.+\left\langle\frac{F_{-1}^{(1)}(t)}{r^{3}} \frac{F_{+1}^{(1)}(0)}{r_{0}^{3}}\right\rangle \exp (i \omega t)\right\} \underset{\text { en av }}{ } d t, \\
\left(R_{2 p, d}\right)^{-1}= & 2^{-1}\left(R_{1 p, d}\right)^{-1} \\
& +3^{-1} \kappa^{2} \int_{0}^{\infty}\left\{\left\langle\frac{F_{0}^{(1)}(t)}{r^{3}} \frac{F_{0}^{(1)}(0)}{r_{0}^{3}}\right\rangle\right\}_{\text {en av }} d t
\end{aligned}
$$

Curly brackets denote a thermal ensemble average with respect to spatial variables, and square brackets denote a trace over the spin variables. Substituting Eq. (8) into Eqs. (9) and (10) gives

$$
\begin{aligned}
\left(R_{1 p, d}\right)^{-1}= & -3^{-1} \kappa^{2}\left(\frac{4 \pi}{5}\right) \sum_{p, p^{\prime}=-1}^{1} c_{+1, p} c_{-1, p^{\prime}} \\
& \cdot \int_{0}^{\infty}\left\{\frac{Y_{+1-p}^{(2)}\left(\theta^{\prime}, \varphi^{\prime} ; t\right)}{r^{3}} \frac{Y_{-1-p^{\prime}}^{(2)}(\theta, \varphi ; 0)}{r_{0}^{3}}\right. \\
& \left.\times\left\langle S_{p}^{(1)}(t) S_{p^{\prime}}^{(1)}(0)\right\rangle\right\}_{\text {en av }} \exp \left(-i \omega_{I} t\right) \\
& +\left\{\frac{Y_{-1-p}^{(2)}\left(\theta^{\prime}, \varphi^{\prime} ; t\right)}{r^{3}} \frac{Y_{+1-p^{\prime}}^{(2)}(\theta, \varphi ; 0)}{r_{0}^{3}}\right. \\
& \left.\times\left\langle S_{p}^{(1)}(t) S_{p^{\prime}}^{(1)}(0)\right\rangle\right\}_{\text {en av }} \exp \left(+i \omega_{I} t\right) d t
\end{aligned}
$$

where $r_{0}$ and $r$ are the interspin distances at $t=0$ and $t$, respectively. Prior slow motion theory and the "decomposition" approximation (see above) have assumed that the motions of spatial and spin variables occur on different time scales so that their time correlation functions can be evaluated separately. The primary objective here is to remove that restriction.

The time correlation functions of the electron spin components can be evaluated by writing the spin operators in the Heisenberg representation, giving

$$
\begin{aligned}
\left\langle S_{p}^{(1)}(t) S_{p^{\prime}}^{(1)}(0)\right\rangle= & \operatorname{Tr}\left[\exp \left(i \hbar^{-1} H_{S}(t) t\right) S_{p}^{(1)}\right. \\
& \left.\times \exp \left(-i \hbar^{-1} H_{S}(t) t\right) S_{p^{\prime}}^{(1)}\right] .
\end{aligned}
$$

where $H_{S}(t)$ is the electron spin Hamiltonian. We consider the situation for $S \geqslant 1$ ions, where $H_{S}$ is a sum of Zeeman, zfs, and hyperfine contributions,

$$
H_{S}(t)=H_{\text {Zeem }}(t)+H_{\mathrm{zfs}}(t)+H_{\mathrm{hf}}(t) .
$$

All terms in general fluctuate stochastically due to the effects of Brownian motion. We neglect the hyperfine term as well as the time-dependent part of $H_{\text {Zeem }}(t)$ (i.e., that due to $g$-anisotropy), since these terms, which can provide important spin relaxation mechanisms for $S=1 / 2$ species, are normally negligible compared to $H_{\mathrm{zfs}}(t)$ for $S \geqslant 1$ ions. Writing the Zeeman term in the laboratory coordinate frame and the zfs interaction in the molecular-fixed zfs-PAS, gives

$$
H_{S}=g \beta_{0} B_{0} S_{0}^{(1)}+\left(\frac{2}{3}\right)^{1 / 2} h c D \hat{S}_{0}^{(2)}+h c E\left(\hat{S}_{+2}^{(2)}+\hat{S}_{-2}^{(2)}\right),
$$

where $D$ and $E$ are the uniaxial and rhombic zfs parameters of esr spectroscopy, $h$ is Planck's constant, and $c$ is the speed of light. Equation (14) is written in mixed coordinate systems. The electron spin operators written without a superscripting caret are defined in the laboratory coordinate system and those with a caret (e.g., $\left.\hat{S}_{q}^{(1)}\right)$ in the molecule-fixed zfs-PAS. The latter can be transformed to the laboratory frame using the Wigner rotation matrices, 


$$
\hat{S}_{p}^{(l)}=\sum_{p^{\prime}=-l}^{+l} S_{p^{\prime}}^{(l)} D_{p^{\prime}, p}^{(l)}(\alpha \beta \gamma),
$$

where $\alpha, \beta, \gamma$ are the Euler angles which effect the transformation (laboratory $\rightarrow$ PAS), giving

$$
\begin{aligned}
H_{S}(t)= & g \beta_{0} B_{0} S_{0}^{(1)} \\
& +\left(\frac{2}{3}\right)^{1 / 2} h c D \sum_{p}\left\{S_{p}^{(2)} D_{p, 0}^{(2)}(\alpha \beta \gamma ; t)\right\} \\
& +h c E \sum_{p}\left\{S_{p}^{(2)} D_{p,+2}^{(2)}(\alpha \beta \gamma ; t)\right. \\
& \left.+S_{p}^{(2)} D_{p,-2}^{(2)}(\alpha \beta \gamma ; t)\right\} .
\end{aligned}
$$

In Eq. (16), the time dependence of $H_{S}(t)$ lies entirely in the Euler angles $(\alpha \beta \gamma)$, which fluctuate due to Brownian reorientational motion. Time dependence can in general also occur in the zfs-parameters $D$ and $E$, which provides a mechanism for electron spin relaxation. This relaxation contribution is not simulated directly but rather is described by the parameter $\tau_{S, v}$ (see Eq. (21 below).

\section{INTRAMOLECULAR RELAXATION}

In this case, the interspin distance $\left(r, r_{0}\right)$ is constant, and the orientation of the $I-S$ vector is fixed in the molecular coordinate frame, specified by the polar angles $(\hat{\theta}, \hat{\varphi})$. Transforming the spherical harmonics in Eq. (11) from the lab to the molecular coordinate frame gives

$$
Y_{q}^{(2)}(\theta \varphi)=\sum_{q^{\prime}} Y_{q^{\prime}}^{(2)}(\hat{\theta} \hat{\varphi}) D_{q^{\prime}, q}^{(2)}(\alpha \beta \gamma)
$$

In the absence of internal molecular motion, the functions $Y_{q^{\prime}}^{(2)}(\hat{\theta} \hat{\varphi})$ are time independent and calculable from a knowledge of the static molecular structure (assuming that the orientation of the zfs-PAS is known). In spin dynamics calculations of intramolecular relaxation, $R_{1 p}$ is evaluated directly from Eqs. (11) [after substitution of (17)], (12), and (16), using an ensemble average of trajectories in which the time dependence of the spin and spatial variables is determined by the motion of the Euler angles $(\alpha \beta \gamma ; t)$.

\section{INTERMOLECULAR RELAXATION}

For intermolecular relaxation, the variables $(\theta, \varphi)$, which define the orientation of the $I-S$ vector in the laboratory frame, are time dependent due to translational diffusion. Their motion is, to a good approximation, uncorrelated with the Euler angles $(\alpha \beta \gamma)$, which specify molecular orientation. Thus the time correlation functions for spin motion,

$$
G_{p}^{S}(t)=\left\{\left\langle S_{p}^{(1)}(0) \cdot S_{-p}^{(1)}(t)\right\rangle\right\}_{\mathrm{en} \mathrm{av}},
$$

and for translational diffusion,

$$
\begin{aligned}
G_{\mathrm{tr}}(t)= & N \int_{\bar{r}} \int_{\bar{r}_{0}} \frac{Y_{q}^{(2)}\left(\theta^{\prime} \varphi^{\prime} ; t\right)}{r^{3}} \frac{Y_{-q}^{(2)}(\theta \varphi ; 0)}{r_{0}^{3}} \\
& \times P\left(\overline{r_{0}} \mid \bar{r}, t\right) d \bar{r} d \overline{r_{0}},
\end{aligned}
$$

where $N$ is the number density of paramagnetic spins, can be computed separately. Various forms are available ${ }^{32-36}$ for $G_{\mathrm{tr}}(t)$. We have used the expression of Torrey ${ }^{32}$ and Abragam $^{34}$ describing translational diffusion of a sphere of diameter $d$, and a mutual diffusion coefficient $D_{12}=\left(D_{1}\right.$ $+D_{2}$ ) for solute/solvent diffusion:

$$
G_{\mathrm{tr}}(t)=\frac{N}{d^{3}} \int_{0}^{\infty}\left[J_{3 / 2}(u)\right]^{2} \exp \left(\frac{-2 D_{12}}{d^{2}} u^{2} t\right) \frac{d u}{u},
$$

where $J_{n}(u)$ is a Bessel function. Alternatively, the translational time correlation function of Ref. 35, which accounts for the effects of excluded volume of the solute and is slightly more accurate than Eq. (20), could have been used. In simulations described below, the integral in (20) was evaluated at discrete time intervals by numerical integration and the results used to construct a look-up table of $G_{\mathrm{tr}}(t)$, which was accessed by interpolation using a cubic spline algorithm.

\section{IMPLEMENTATION OF SPIN DYNAMICS SIMULATIONS}

The time correlation functions of Eq. (11) were evaluated numerically by constructing an ensemble of trajectories (typically several hundred) of the time dependence of the spin operators and spatial functions. Each spin trajectory was computed by direct numerical evaluation of Eqs. (12) and (16), where the time dependence lies in the Euler angles of Eq. (16). The starting Euler angles were defined by the 92 vectors from the origin to the 32 faces and 60 vertices of a truncated icosahedron (buckeyball). Molecular reorientational diffusion was assumed to occur in discrete step with a uniform step interval $\tau_{r}$. At each reorientational step, the molecular coordinate frame was rotated about an axis orientated randomly in the laboratory frame, with the rotation angle chosen as a random Gaussian deviate of standard deviation $\sigma_{j}$ from mean zero. The time interval $\tau_{r}$ between reorientational steps was set equal to $\tau_{R}^{(1)} / n_{r}$ with $n_{r}=30$ in most simulations $\left(\tau_{R}^{(l)}\right.$ is the reorientational correlation time of an $l$ th rank molecule-fixed tensor; $\tau_{R}{ }^{(l)}=\tau_{R}^{(1)} / l(l+1)$ where $\tau_{R}^{(1)}$ is Debye's correlation time for a molecule-fixed vector). This value of $n_{r}$ was found in trial calculations to provide a conservative simulation of the small-step diffusion limit (see below). The following relation, which was determined by numerical simulation, exists between $n_{r}$ and $\sigma_{j}$ :

$$
\sigma_{j}=\pi \sqrt{0.3037 / n_{r}} .
$$

In calculations, $n_{r}$ was first set to the desired number of reorientational steps per $\tau_{R}^{(1)}$ interval, and $\sigma_{j}$ was calculated. At successive reorientational steps, the jump angle and rotation axis were selected randomly as described above, and the Wigner rotation matrix elements were calculated and used to rotate the molecule-fixed coordinate axes from the $n$th to the $(n+1)$ st orientation. Then the Euler angles, the Wigner rotation matrix elements needed in Eqs. (16) and (17), and the new spin Hamiltonian corresponding to the $(n+1)$ st point 
were evaluated. The spin operators were propagated across the $n \rightarrow(n+1)$ time interval using the Hamiltonian $H_{S}(n)$.

The spin components $S_{q}(t)(q=x, y, z)$ were computed at discrete time intervals, $n \tau_{\text {prop }}$, where $n$ is integral and $\tau_{\text {prop }} \leqslant \tau_{r}$. To describe the precessional motion accurately, it is important that $S_{q}(t)$ be computed at several time points per cycle of the highest frequency components of the precessional motion. Cubic spline interpolation provides an accurate representation of a sine wave when the sampling frequency is at least 6 points per cycle. ${ }^{37}$ Thus the sampling interval $\tau_{\text {prop }}$ was set to be no more than one-sixth the period of the highest frequency components of $H_{S}$ : specifically, $\tau_{\text {prop }}=\left(2 \pi / 7 H_{i j}\right)$, where $H_{i j}$ was the largest matrix element of $H_{S}$, was used in most calculations. This choice of sampling frequency ensured both convergence of the propagator and an accurate, though relatively sparse, representation of the precessional motion. The sampling interval $\tau_{\text {prop }}$, after calculation in this way, was adjusted slightly (when necessary) so that the reorientational step time $\tau_{r}$ was an integral multiple of the sampling interval: $n_{\text {prop }} \tau_{\text {prop }}=\tau_{r}$.

The propagator $P_{n}=\exp \left(i H_{S}(n) t\right)$ was computed after each reorientational step by direct evaluation of its defining series using the newly evaluated Hamiltonian. Successive terms of the exponential series were computed and coadded until the computed term was smaller than the bit size in double precision (at around $10^{-18}$ ), which occurred typically after the 9 th-14th term. Provision was made to report convergence failure, although the algorithm is quite robust when $\tau_{\text {prop }}$ is chosen as described above. Numerical propagation of the spin operators by repeated matrix multiplication can produce a loss of norm of the propagated operators over prolonged trajectories. This was found to be a serous problem in single precision but not in double precision for trajectories involving several thousand steps.

After calculating the propagation step interval $\tau_{\text {prop }}$ as described above, $\tau_{\text {prop }}$ was further shortened if necessary so that $\tau_{\text {prop }} \leqslant\left(\tau_{S} / 10\right)$ and $\tau_{\text {prop }} \leqslant\left(\tau_{D} / 10\right)$, where $\tau_{S}$ is the electron spin relaxation time and $\tau_{D}=a^{2} / D_{12}$ is the correlation time for translational diffusion. In summary, $\tau_{\text {prop }}$ was in general set equal to the shortest of the four quantities, $\left(2 \pi / 7 H_{i j}\right),\left(\tau_{R}^{(1)} / 30\right),\left(\tau_{S} / 10\right)$, and $\left(\tau_{D} / 10\right)$, thereby ensuring an accurate representation of the spin trajectory with respect to precessional motion, reorientational motion, spin relaxation, and translational diffusion.

The length of the trajectory was chosen to be long enough that the dipolar time correlation functions decayed to a small fraction (usually $<1 \%$ ) of their initial values. This was done by choosing the number of propagation steps sufficiently large that the length of the trajectory was equal to the smallest of the following quantities: $5 \tau_{S, v}, 5 \tau_{R}^{(1)}$, or (for intermolecular relaxation) $10 \tau_{D}$.

Trajectories comprise at least 50, and typically several hundred time points, at each of which the dipolar time correlation function was calculated. Then the ensemble average of Eq. (11) was constructed as a sum of 92 such trajectories (more if desired). The integral was evaluated using a cubic spline interpolation algorithm. ${ }^{37}$ Calculations of individual $R_{1 p}$ values for a spin $S=1$ using an ensemble of 92 trajec-

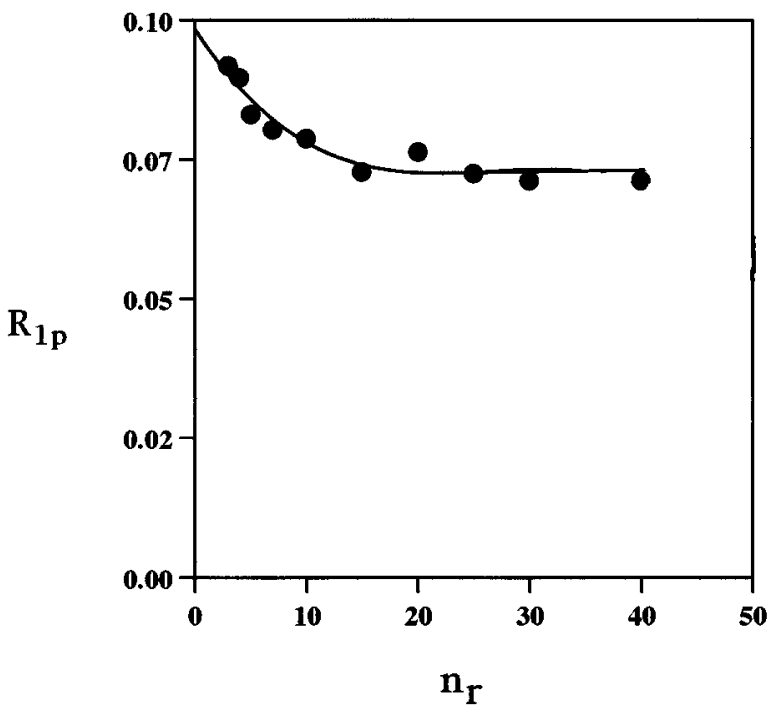

FIG. 1. Dependence of simulated $R_{1 p}$ values on the reorientational step size. $n_{r}$ is the number of reorientational steps per $\tau_{R}^{(1)}$. Parameters as given in the legend of Fig. 6, except $\tau_{R}^{(1)}=1.0 \times 10^{-10} \mathrm{~s}$.

tories, each containing 600 time points, required approximately 1 min using a 7100/80 (80 MHz) PowerMac computer. The code was written for the Language Systems Fortran-77 compiler for the PowerPC. The program (SpinDyn.f) should be portable to other Fortran compilers and can be supplied upon request.

As stated above, calculations were performed to examine the effect of reorientational step size (as specified by the parameter $n_{r}$ ) on the simulation (Fig. 1). For this purpose, conditions were assumed under which $R_{1 p}$ is relatively sensitive to molecular reorientation (see figure legend). Simulated $R_{1 p}$ values were found to be approximately independent of $n_{r}$ for $n_{r}>10$. Other simulations of this study used $n_{r}=30$, which corresponds conservatively to the small-step diffusional limit.

\section{ELECTRON SPIN RELAXATION}

Electron spin relaxation for spin $S \geqslant 1$ ions arises principally from thermal modulation of the zfs tensor, ${ }^{38}$ which can result both from Brownian reorientational motions ${ }^{39,40}$ and from collisionally induced vibrational damping of the ion coordination sphere. ${ }^{41-44}$ The correlation times, $\tau_{R}^{(2)}$ and $\tau_{v}$, for these two processes are very different, typically $\tau_{R}^{(2)}>50$ ps vs $\tau_{v}<5$ ps in low molecular weight metal complexes, and thus the reorientational and vibrational degrees of freedom can, to a good approximation, be treated independently. Accordingly, we write the electron spin relaxation rate $\tau_{S}^{-1}$ as a sum of contributions,

$$
\tau_{S}^{-1}=\tau_{S, r}^{-1}+\tau_{S, v}^{-1}
$$

due, respectively, to the reorientational and vibrational (or distortional) modulation of the electron spin Hamiltonian. For $S \geqslant 1$ ions, it is usual that $\tau_{R}^{(2)}>\tau_{S, r}$, so that the reorientational contribution $\tau_{S, r}^{-1}$ is outside the Redfield limit. $\tau_{v}$ 
describes a process that is physically related to vibrational relaxation and has a magnitude ranging, according to molecular dynamics simulations ${ }^{29,30,45}$ and IR linewidth measurements, ${ }^{42}$ from some tens of femtoseconds up to a few picoseconds. Because $\tau_{v} \ll \tau_{R}^{(2)}$, it is often the case that for $S \geqslant 1$ ions, $\tau_{S, v}$ can be described by the Redfield theory, ${ }^{39}$ while $\tau_{S, r}$ cannot.

The algorithms of SpinDyn.f carry out a first principles calculation of $\tau_{S, r}^{-1}$, which is not subject to the limitations of the Redfield theory, and which is physically appropriate within the framework of the phenomenological static spin Hamiltonian of Eq. (14) and the isotropic reorientational diffusion model described above. No parameters $\tau_{S, r}$ need be assumed. On the other hand, a vibrational relaxation time $\tau_{S, v}$ is required as a parameter of the calculation. A first principles simulation of $\tau_{S, v}$ has recently been undertaken as part of a study of the hexaaqua $\mathrm{N}_{1}$ (II) ion. ${ }^{29,30}$ This simulation required a quantum mechanical calculation of the derivatives of the zfs tensor with respect to the normal vibrational coordinates, as well as a molecular dynamics simulation of the collisionally induced vibrational relaxation processes. The calculations are complex and of limited accuracy, and we have not attempted to incorporate a simulation of this type into SpinDyn.f. Rather, a field dependent relaxation parameter $\tau_{S, v}$ of the form derived by McLachlan ${ }^{39}$ was used:

$$
\begin{aligned}
& \left\langle\frac{1}{\tau_{S, v}}\right\rangle=\frac{1}{\tau_{S, v}^{(0)}}\left(\frac{0.2}{1+\omega^{2} \tau_{v}^{2}}+\frac{0.8}{1+4 \omega^{2} \tau_{v}^{2}}\right), \\
& \frac{1}{\tau_{S, v}^{(0)}}=\left(\Delta^{2} / 5\right)\{4 S(S+1)-3\} \tau_{v}, \\
& \Delta^{2}=(2 \pi c)^{2}\left[(2 / 3)\left(D^{\prime}\right)^{2}+2\left(E^{\prime}\right)^{2}\right] .
\end{aligned}
$$

$\omega$ is the level splitting, $c$ is the speed of light, and $\Delta^{2}$ is the trace of the square of the dynamic part of the zfs tensor for which zfs parameters are $D^{\prime}$ and $E^{\prime}$. An analogous set of expressions to Eqs. (22)-(24) can be used to describe $\left\langle\tau_{S, r}^{-1}\right\rangle$ when the Redfield theory is appropriate, i.e., when $\tau_{S, r} \gg \tau_{R}^{(2)}$. In this case, $\Delta^{2}$ is the trace of the square of the static zfs tensor (i.e., $D^{\prime}=D, E^{\prime}=E$ ), and the correlation time is $\tau_{R}^{(2)}$. For spins $S>1$ outside the extreme narrowing limit, electron spin relaxation is in general a multiexponential process for which Eq. (22) represents a weighted average of rate constants. A recent detailed analysis ${ }^{46}$ of nonexponential spin relaxation for $S=5 / 2$ in the Redfield limit indicates that corrections to Eq. (22) are small, on the order of a few percent or less.

In the simulations described below we have elected to fix $\tau_{S, v}$ at its low field value $\tau_{S, v}^{(0)}$, i.e., to assume $\omega \tau_{v} \ll 1$ in Eq. (22). We have done this because it has seemed better, for the purpose of understanding the nature of reorientationally induced deviations from the analytical theory, to study first the behavior of the NMR-PRE when $\tau_{S, v}$ is field independent without injecting an arbitrary parameter $\tau_{v}$ into the analysis. Simulations performed with $\tau_{v} \rightarrow 0$ and variable $\tau_{S, v}^{(0)}$ have

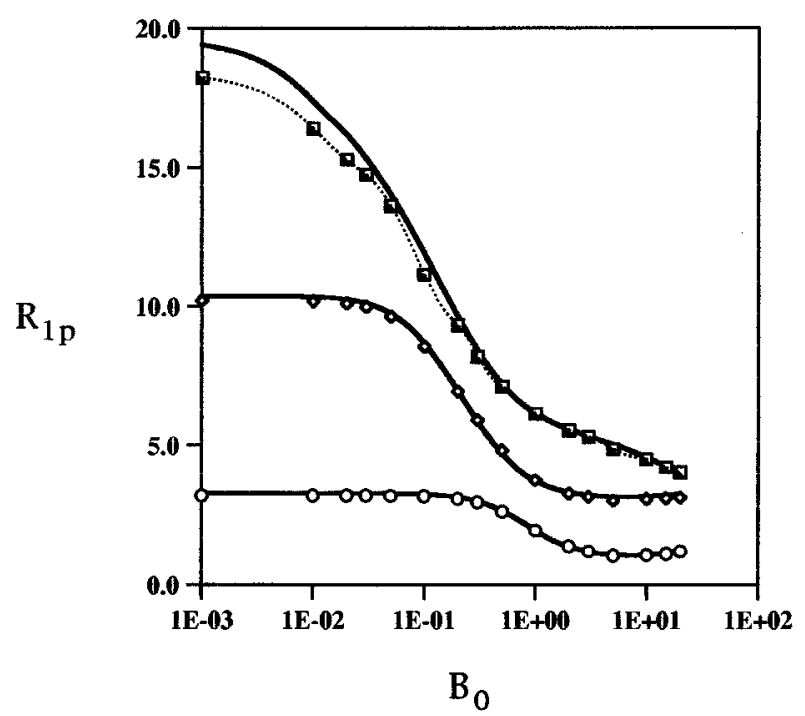

FIG. 2. Comparison of the spin dynamics calculations (symbols, dashed curve), with results from the intermolecular analytical Zeeman-limit theory (solid curves) for $S=1$ assuming zero static zfs interaction $(D, E=0)$. Molecular self diffusion coefficients of the solvent and solute were taken to be $D_{1}=1.4 \times 10^{-9} \mathrm{~m}^{2} \mathrm{~s}^{-1}$ and $D_{2}=4.1 \times 10^{-10} \mathrm{~m}^{2} \mathrm{~s}^{-1}$, respectively, and the distance of closest approach was $d_{c}=0.4 \mathrm{~nm}$. The concentration of electron spins was $0.020 \mathrm{M}$. The three pairs of curves in Fig. 2 show the effect of decreasing electron spin relaxation time on the field dispersion profiles of the intermolecular $R_{1 p}$. In this series, $\tau_{S, v}^{(0)}$ values were (a) $1.0 \times 10^{-1} \mathrm{~s}$ (squares), (b) $1.0 \times 10^{-10} \mathrm{~s}$ (diamonds), and (c) $1.0 \times 10^{-11} \mathrm{~s}$ (circles), with $\tau_{v}$ very short $\left(10^{-13} \mathrm{~s}\right)$.

provided a basis for understanding the qualitative effects to be expected when $\tau_{S, v}$ is field dependent. These effects are discussed further below.

\section{PERFORMANCE OF THE SPIN DYNAMICS SIMULATION}

The performance of the program SpinDyn.f has been tested with respect to its behavior in the Zeeman and zfs limits, where analytical theory is available for comparison. Figure 2 compares the results of Zeeman-limit $(D, E=0) \mathrm{SD}$ simulations (symbols) against results computed using analytical Zeeman-limit theory (solid curves). The calculations assumed the slow motion limit $\left(\tau_{R}^{(1)} \rightarrow \infty\right)$, with three values of $\tau_{S, v}^{(0)}: \tau_{S, v}^{(0)}=10^{-1} \mathrm{~s}, 10^{-10} \mathrm{~s}$, and $10^{-11} \mathrm{~s} . \tau_{v}$ was taken to be very short, $\tau_{v}=10^{-13} \mathrm{~s}$, as described above. The SD simulations of Fig. 2 were computed from ensembles consisting of $6 \times 92=552$ averaged trajectories. This level of averaging produced very little scatter in the calculations and required about 8 min per point using a PowerMacintosh 7100.

The SD results were generally in quite good agreement with Zeeman-limit analytical theory (Fig. 2). For some calculations with $B_{0}$ small and both $\tau_{S, v}$ and $\tau_{R}^{(1)}$ very long, the SD calculations fell a few percent below the analytical theory (Fig. 2, top). This divergence occurred because under these conditions, which are not encountered in practice, the SD algorithm prematurely truncates the long-time tail of $G_{\mathrm{tr}}(t)$. It is well known that translational time correlation 


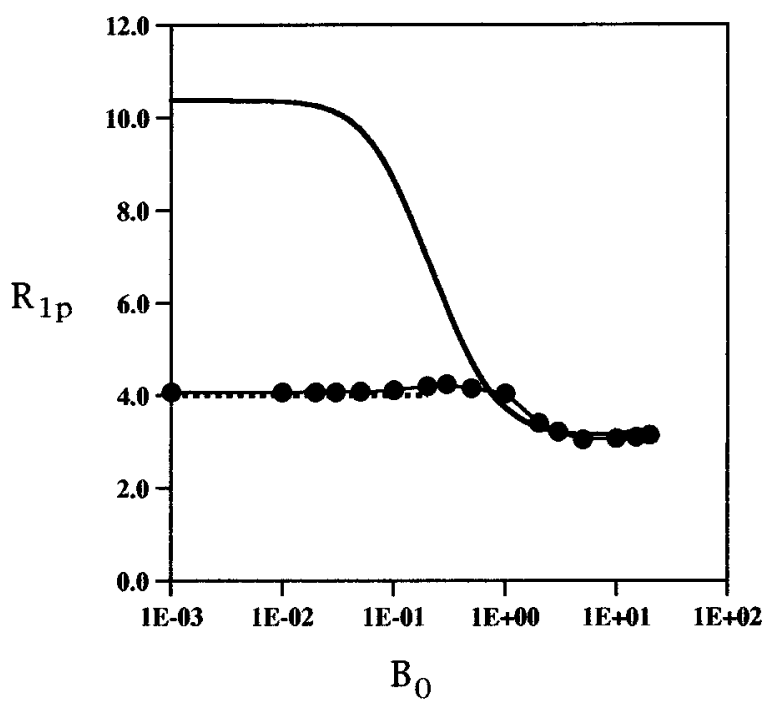

FIG. 3. Comparison of the spin dynamics calculations (circles), with calculations based on the limiting analytical expressions of intermolecular Zeeman-limit theory (solid line) and zfs-limit theory (dashed line) assuming the uniaxial zfs interaction with $D=1.0 \mathrm{~cm}^{-1}(E=0)$. Molecular reorientation of the solute was assumed to be slow $\left(\tau_{R}^{(1)}=0.1 \mathrm{~s}\right)$. Other parameters are given in the legend of Fig. 2, except $\tau_{S, v}^{(0)}=1 \times 10^{-10} \mathrm{~s}$.

functions fall off with interspin distance much more slowly than do the time correlation functions for intramolecular reorientation. Physically, this behavior results from the contributions of $I-S$ spin pairs at relatively large interspin distances, because the number of contributing dipolar couplings increases as $r^{2}$ and because the effective correlation time for intermolecular dipolar coupling increases as $r^{2} / D$. Thus $I-S$ spin pairs at large distances contribute disproportionately to the relaxation process, so that the interspin distance dependence of intermolecular relaxation is much milder than the $r^{-6}$ dependence of intramolecular relaxation. Computationally, the long time tail of $G_{\mathrm{tr}}(t)$ becomes important when both $\tau_{R}^{(1)}$ and $\tau_{S, v}$ are long, a situation that is a bit difficult to simulate because of the large number of translational steps needed. Experimentally, however, for $S \geqslant 1$ ions, $\tau_{S, v}$ is always sufficiently short to mask contributions of the longtime tail of $G_{\mathrm{tr}}(t)$, so that this does not represent a practical limitation of the program.

Figure 3 compares a simulated field dispersion profile (fdp) (symbols) for the intermolecular $R_{1 p}$ when the zfs interaction is nonzero, with fdp's computed using the analytical theories. The simulation was performed under slow motion conditions $\left(\tau_{R}^{(1)} \rightarrow \infty\right)$ and should agree with analytical zfs-limit theory (dashed line) in the low field region and with the analytical Zeeman-limit theory (solid line, no symbols) in the high field region. An electron spin $S=1$ subject to a uniaxial zfs interaction $\left(D=1 \mathrm{~cm}^{-1}, E=0\right)$ was assumed (see legend of Fig. 2 for other conditions); for a zfs coupling of this magnitude, the intermediate regime where the precessional quantization axis changes from the molecule-fixed zfsPAS to the laboratory frame occurs at magnetic field strengths in the vicinity of $B_{0}=1 T$. As is evident in the figure, the spin dynamics simulations were in good agree-

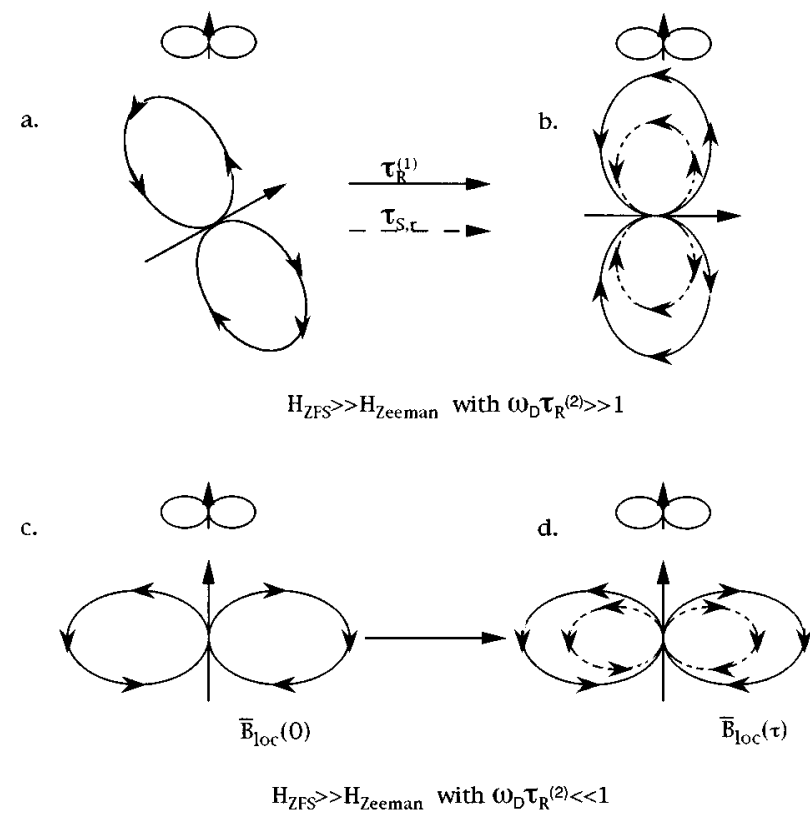

FIG. 4. Schematic depiction of the origins of time dependence in the electron-nuclear (SI) dipole-dipole coupling interaction when $H_{z \mathrm{fs}}$ $\gg H_{\text {Zeem }}$. (a) and (b) describe the situation for zfs-type precession (precession quantized along molecule-fixed axes, which occurs when $\omega_{D} \tau_{R}^{(2)} \gg 1$ ); (c) and (d) describe Zeeman-type precession (quantized along the external magnetic field, occurring when $\left.\omega_{D} \tau_{R}^{(2)} \ll 1\right)$. Panels on the left and right illustrate schematically the time dependence in the average local dipolar magnetic field, $\langle\bar{B}(\Omega ; t)\rangle$, associated with $S_{z}$ that is produced by Brownian reorientation through a specified set of Euler angles $\Omega$ over a time interval $t$. In the presence of zfs-type precession [(a) and (b)], $\langle\bar{B}(\Omega ; t)\rangle$ fluctuates due both to the change in quantization axis of $S$ (solid flux lines), and due to spin relaxation caused by stochastic motion of the zfs-PAS (dashed flux lines). In the presence of Zeeman-type precession [(a) and (b)], $\bar{S}$ does not reorient, but relaxation of $\bar{S}$ resulting from reorientational modulation of the zfs tensor causes a decrease in $\langle\bar{B}(\Omega ; t)\rangle$.

ment with the results of analytical theory in both the zfs and Zeeman limits.

\section{EFFECT OF BROWNIAN REORIENTATION ON THE FIELD DISPERSION PROFILE}

Reorientational effects on the fdp operate through four rather distinct physical mechanisms when the physical situation is outside the Zeeman limit, and this complexity can lead to some confusion in discussion. For purposes of clarity, three of the four mechanisms are illustrated schematically in Fig. 4, which shows the spin vectors and flux lines of the local dipolar magnetic fields due to $S_{z}$ and $I_{z}$ at an arbitrary initial time $t=0$ and at a later time $t=\tau$. Flux lines of the transverse components of $\bar{S}\left(S_{x, y}\right)$ are not shown in the figure since the rapid spin precession of $\bar{S}$ tends to decouple these fields from the motion of $\bar{I}$ and makes them relatively ineffective as agents of nuclear spin relaxation (although this approximation breaks down when zfs rhombicity is large and/or electron spin relaxation is extremely rapid).

Figures 4(a) and 4(b) illustrate the zfs-limit situation $\left(H_{\mathrm{zfs}} \gg H_{\text {Zeem }}\right)$ when $\left(\tau_{R}^{(2)} \omega_{D} \gg 1\right)$. In this case, molecular reorientation is slow enough that the precession of $\bar{S}$ is quan- 


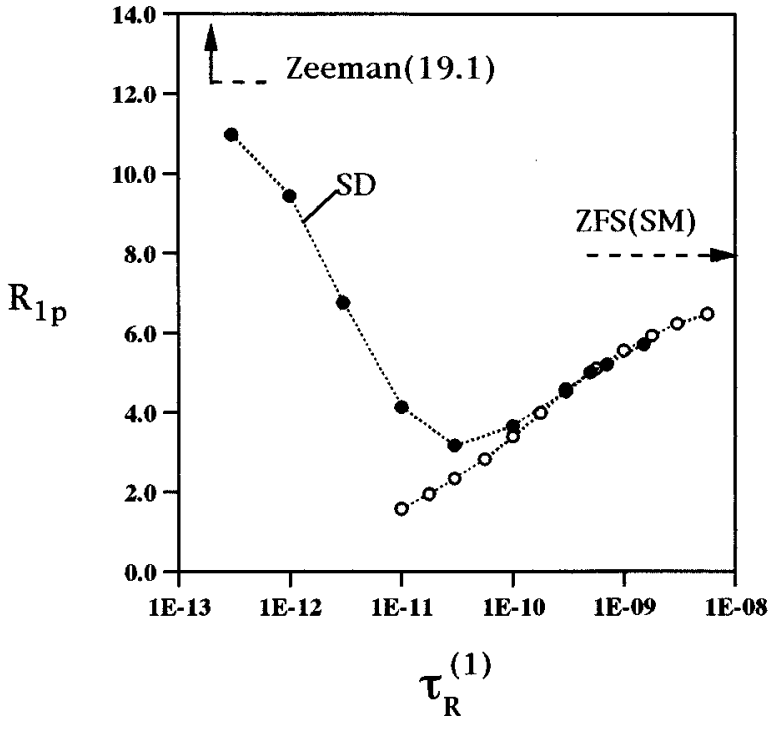

FIG. 5. $R_{1 p}$ as a function of reorientational correlation time $\tau_{R}^{(1)}$ for $S=1$ assuming a uniaxial zfs interaction of $D=1.0 \mathrm{~cm}^{-1}(E=0.0)$, and a Zeeman field, $B_{0}=10^{-5}$ T. Comparison of results obtained from SD simulations (filled circles) and intermolecular zfs-limit analytical theory (open circles). The slow motion zfs-limit (ZFS(SM)) result is shown as a short dashed line with an arrow. The zfs-limit analytical theory was calculated using a $\tau_{d d}^{-1}$ correlation time of the form of Eq. (25) and $\tau_{S, r}^{-1}$ the form of Eq. (26). Other conditions are given in the legend of Fig. 2.

tized in the molecule-fixed coordinate frame. Figures 4(c) and 4(d) illustrate the zfs-limit situation $\left(H_{\mathrm{zfs}} \gg H_{\text {Zeem }}\right)$ when $\left(\tau_{R}^{(2)} \omega_{D} \ll 1\right)$, in which case the precessional motion of $\bar{S}$ is reorientationally decoupled from the zfs interaction and becomes Zeeman-like in character. In both cases, the precession of $\overline{\boldsymbol{I}}$ is quantized along the external magnetic field $\bar{B}_{0}$. Figures 4(b) and 4(d) illustrate the local field of $S_{z}$ at $t=\tau$ following Brownian reorientation through a specified set of Euler angles $(\alpha \beta \gamma)$. In Fig. 4(b) (zfs-type precession), molecular reorientation alters the orientation of the PAS of $\bar{B}_{\text {loc }}(t)$ and hence the spatial angles of the dipole-dipole interaction, as indicated by the solid flux lines. In addition, stochastic time dependence in the zfs Hamiltonian due to Brownian reorientation produces electron spin relaxation, as described in Eq. (21) by the parameter $\tau_{S, r}$. The effects of $\tau_{S, r}$ in decreasing the average value of $\bar{B}_{\text {loc }}(\tau)$ are described schematically by the dashed flux line in the figure. In addition to these two effects, reorientation also alters the precessional frequencies of $\bar{S}$ when $H_{\mathrm{zfs}} \approx H_{\mathrm{Zeem}}$. However, this third effect occurs only in the intermediate regime and even then should be small since only the transverse components $S_{x, y}$ are involved; fields of precessing transverse spin components are not depicted in Fig. 4.

Figures 4(c) and 4(d) illustrate the "zfs-limit" ( $H_{\mathrm{zfs}}$ $\left.\gg H_{\text {Zeem }}\right)$ when reorientation is fast enough that $\omega_{D} \tau_{R}^{(2)} \ll 1$, in which case molecular reorientation decouples the zfs interaction from the spin precessional motion, which then has Zeeman character (even though $H_{\mathrm{zfs}} \gg H_{\mathrm{Zeem}}$ ). $H_{\mathrm{zfs}}$ then influences $R_{1 p}$ only as an agent of electron spin relaxation $\left(\tau_{S, r}\right)^{-1}$. In this situation, where the zfs interaction is large

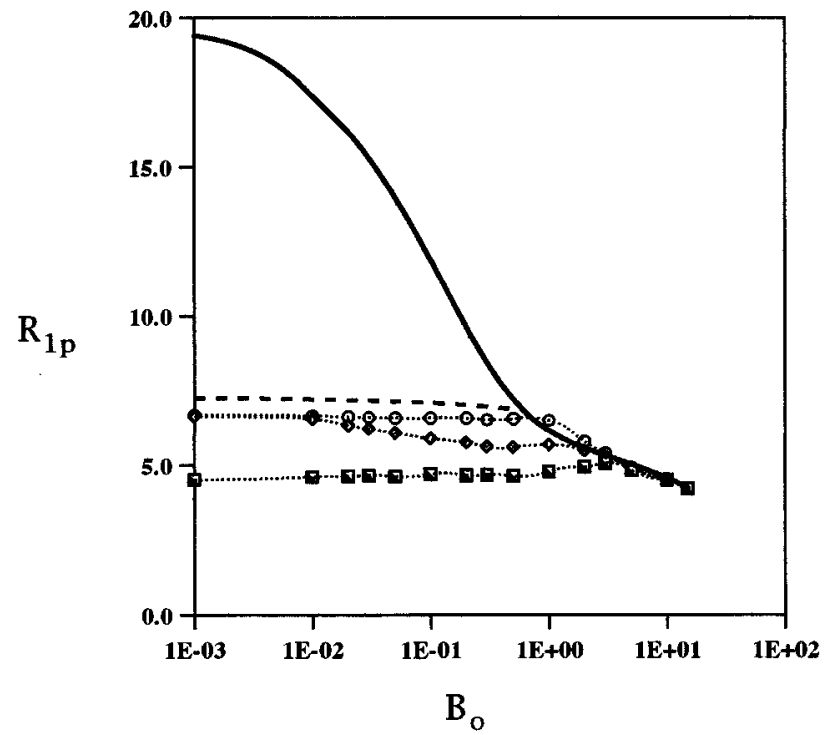

FIG. 6. Comparison of spin dynamics simulations (symbols) with limiting analytical expression for zfs-limit theory (bold dashed line) and the Zeemanlimit theory (solid line), assuming a uniaxial zfs interaction with $D=1.0$ $\mathrm{cm}^{-1}(E=)$. Simulated curves 0 who the effect of varying the solute $\tau_{R}^{(1)}$ on the field dispersion profiles of the intermolecular $R_{1 p} . \tau_{R}^{(1)}$ values are (a) $1.0 \times 10^{-8} \mathrm{~s}$ (circles), (b) $1.0 \times 10^{-9} \mathrm{~s}$ (diamonds), (c) $3.0 \times 10^{-10} \mathrm{~s}$ (squares), with $\tau_{S, v}^{(0)} \rightarrow \infty$. Other physical parameters are given in the legend of Fig. 2.

but is reorientationally decoupled from the precessional motion, the terms "zfs-limit" and "Zeeman-limit" are somewhat ambiguous and should probably be avoided.

Figure 5 shows the dependence of $R_{1 p}$ on molecular reorientation $\left(R_{1 p}\right.$ vs $\left.\tau_{R}^{(1)}\right)$ when $H_{\mathrm{zfs}} \gg H_{\text {Zeem }}$. In these calculations, the polarizing magnetic field strength was assumed to be low enough $\left(B_{0}=10^{-5} \mathrm{~T}\right)$ to ensure that $H_{\mathrm{zfs}}$ $\gg H_{\text {Zeem }}$. The calculations assumed a uniaxial zfs interaction of $D=1 \mathrm{~cm}^{-1}\left(\omega_{D}=1.88 \times 10^{11} \mathrm{rad}_{-\mathrm{s}^{-1}}\right)$, for which the zfsdecoupling condition $\left(\tau_{R}^{(2)} \omega_{D}<1\right)$ occurs in the vicinity of $\tau_{R}^{(2)} \leqslant 5$ ps (or $\tau_{R}^{(1)} \leqslant 15 \mathrm{ps)}$. The results of SD simulations (filled circles) are compared with calculations based on the analytical intermolecular zfs-limit theory of Ref. 5 (open circles). Also shown in Fig. 5 are results of the Zeeman-limit calculation and the slow motion zfs-limit calculation (dashed lines). $R_{1 p}$ is expected to approach the slow motion zfs-limit result $[\mathrm{ZFS}(\mathrm{SM})]$ when $\tau_{R}^{(1)}$ is long, and the Zeeman-limit result when $\tau_{R}{ }^{(1)} \rightarrow 0$. The results of SD simulations were consistent with this behavior, although simulations near the limits $\tau_{R}^{(1)} \rightarrow 0$ and $\tau_{R}^{(1)} \rightarrow \infty$ are difficult to perform because of the large number of steps involved and were not undertaken.

The analytical zfs-limit theory is in good agreement with the results of SD simulations when $\tau_{R}^{(1)}$ is long enough to ensure zfs-type precession (Fig. 5). In this regime, $R_{1 p}$ decreases with decreasing $\tau_{R}^{(1)}$ due to increasingly efficient randomization of the dipolar interaction. The effects of reorientational decoupling of the zfs interaction appear in the vicinity of $\tau_{R}^{(1)} \sim 15 \mathrm{ps}$, where $R_{1 p}$ passes through a minimum; at shorter $\tau_{R}^{(1)}, R_{1 p}$ increases as expected. When 


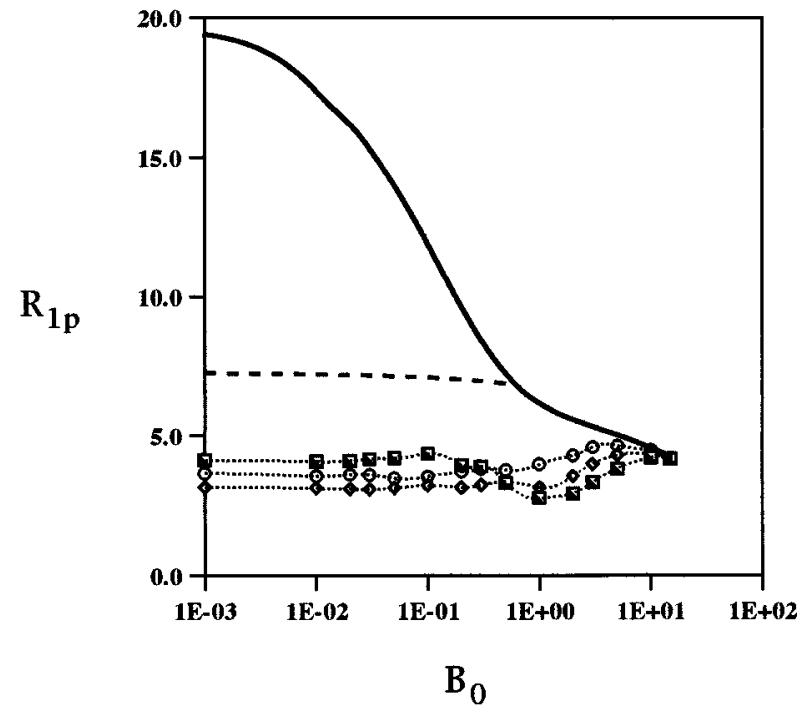

FIG. 7. Comparison of spin dynamics simulations (symbols) with limiting analytical expressions for zfs-limit theory (bold dashed line) and Zeemanlimit theory (solid line). Conditions are as described in the legend of Fig. 6 except for $\tau_{R}^{(1)}$ values, which (a) $3.0 \times 10^{-1} \mathrm{~s}$ (circles), (b) $3.0 \times 10^{-11} \mathrm{~s}$ (diamonds), and (c) $1.0 \times 10^{-11} \mathrm{~s}$ (squares).

$\tau_{R}^{(1)} \rightarrow 0$, the electron spin is completely reorientationally decoupled from the zfs interaction and $R_{1 p}$ is expected to approach the Zeeman-limit value. The simulations are consistent with the expected behavior, although calculations with $\tau_{R}^{(1)}<3 \times 10^{-13} \mathrm{~s}$ were not performed because of the large number of reorientational steps involved.

Figures 6-8 illustrate the influence of molecular reorientational motion on the simulated field dispersion profiles (fdps) of the intermolecular $R_{1 p}$ for an electron spin $S=1$

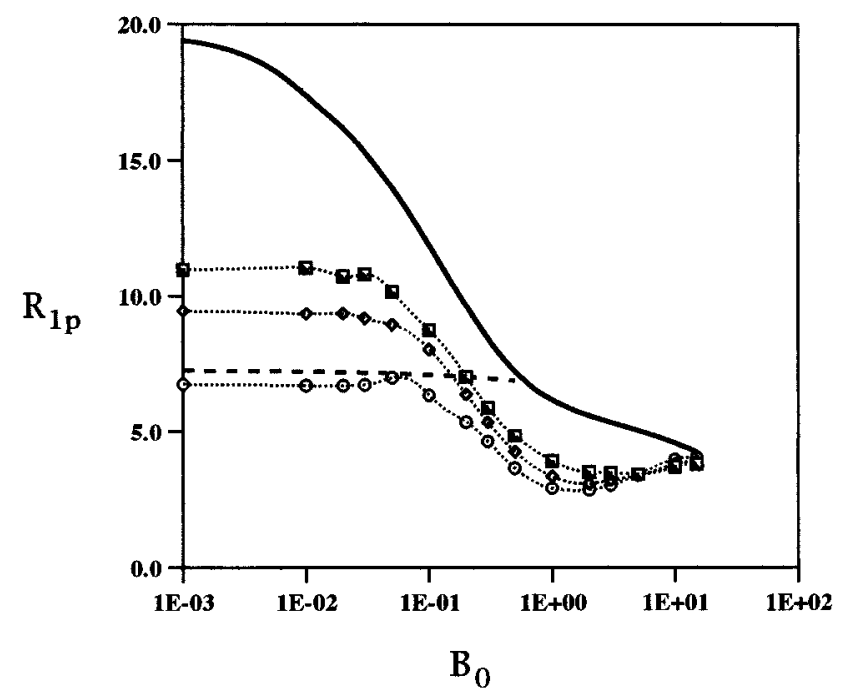

FIG. 8. Comparison of spin dynamics simulations (symbols) with limiting analytical expressions for zfs-limit theory (bold dashed line) and Zeemanlimit theory (solid line). Conditions are as described in the legend of Fig. 6 except for $\tau_{R}^{(1)}$ values, which were (a) $3.0 \times 10^{-12} \mathrm{~s}$ (circles), (b) 1.0 $\times 10^{-12} \mathrm{~s}$ (diamonds), and (c) $1.0 \times 10^{-13} \mathrm{~s}$ (squares). precessing under the influence of a Zeeman interaction plus a uniaxial zfs interaction $\left(D=1.0 \mathrm{~cm}^{-1}, E=0\right)$. The results of analytical theory are shown as lines without symbols: the Zeeman-limit fdp is shown as a solid curve, and the slow motion zfs-limit value of $R_{1 p}$ as a bold dashed line. The simulated curves in these figures correspond to a series of specified $\tau_{R}^{(1)}$ values, ranging from a maximum of $1 \times 10^{-8} \mathrm{~s}$ in Fig. 6 to a minimum of $0.3 \times 10^{-12} \mathrm{~s}$ in Fig. 8. As in Fig. 3 , the intermediate regime $\left(H_{\text {Zeem }} \approx H_{\mathrm{zfs}}\right)$ occurs in the vicinity of $B_{0}=1 T$. Because the effects of molecular reorientation tend to be masked by rapid electron spin relaxation (short $\tau_{S, v}$ ), this set of simulations assumed $\tau_{S, v} \rightarrow \infty$.

Figure 6 shows simulated fdps in the regime of relatively slow molecular reorientation $\left(\omega_{D} \tau_{R}^{(2)} \gg 1\right)$. In this situation, which corresponds to points to the right of the $R_{1 p}$ minimum of Fig. 5, the electron spin undergoes zfs-type precession in the low field regime $\left(B_{0} \ll 1 T\right)$ and Zeeman-type precession in the high-field regime $\left(B_{0} \gg 1 T\right)$. The three simulated curves correspond to $\tau_{R}^{(1)}=1 \times 10^{-8} \mathrm{~s}$ (open circles), $\tau_{R}^{(1)}$ $=1 \times 10^{-9} \mathrm{~s}$ (diamonds) and $\tau_{R}^{(1)}=3 \times 10^{-10} \mathrm{~s}$ (squares). Increasingly rapid Brownian reorientation acts to depress the NMR-PRE in the zfs limit $\left(H_{\mathrm{zfs}} \gg H_{\text {Zeem }}\right)$ and intermediate regimes, clearly a result of reorientational randomization of the dipolar interaction, as depicted schematically in Figs. 4(a) and 4(b). In the Zeeman-limit regime $\left(B_{0} \gg 1 T\right)$, the simulated curves converge to the Zeeman-limit curve of analytical theory, and $R_{1 p}$ becomes independent of solute reorientation.

Figure 8 shows simulated fdps in the regime of fast molecular reorientation $\left(\omega_{D} \tau_{R}^{(2)} \ll 1\right)$, where the zfs interaction is reorientationally decoupled from the spin precessional motion (this regime corresponds to points to the left of the $R_{1 p}$ minimum in Fig. 5). Fdps in Fig. 8 are plotted for $\tau_{R}^{(1)}$ $=3 \times 10^{-12} \mathrm{~s}$ (circles), $\tau_{R}^{(1)}=1 \times 10^{-12} \mathrm{~s}$ (diamonds), and $\tau_{R}^{(1)}=0.3 \times 10^{-13} \mathrm{~s}$ (squares). The low field limiting value of $R_{1 p}$ rises as $\tau_{R}^{(1)}$ falls, apparently approaching the Zeemanlimit value as $\tau_{R}^{(1)} \rightarrow 0$, as occurs in Fig. 5. Figure 7 shows simulated fdps calculated assuming $\tau_{R}^{(1)}$ values near the $R_{1 p}$ minimum of Fig. 5, specifically, $\tau_{R}^{(1)}=1 \times 10^{-10} \mathrm{~s}$ (circles), $\tau_{R}^{(1)}=3 \times 10^{-11} \mathrm{~s}$ (diamonds), and $\tau_{R}^{(1)}=1 \times 10^{-11}$ $\mathrm{s}$ (squares).

A different way of illustrating the phenomenon of reorientational decoupling of electron spin precession from the zfs interaction is to vary the zfs parameter $D$ at fixed $\tau_{R}^{(1)}$ and fixed polarizing field strength $B_{0}$. This is shown in Fig. 9, where $R_{1 p}$ is plotted vs $D^{2}$ with $\tau_{R}^{(1)}=30 \mathrm{ps}$. When the condition for zfs-type precession is satisfied $\left(\omega_{D} \tau_{R}^{(2)}\right.$ $\gg 1$, or $D \gg 0.5 \mathrm{~cm}^{-1}$ in Fig. 9), $R_{1 p}$ becomes independent of $D$. However, it is important to note this conclusion holds specifically for a uniaxial zfs interaction-the dependence of the fdp on the zfs $E$-term, when present and significant, is profound, often much greater than the dependence on the $D$-term. ${ }^{12,13}$

Finally, it should be noted that the simulations of Figs. 6-8 assumed that $\tau_{S, v}$ was very long $(0.1 \mathrm{~s})$ in order to emphasize the nature of the influence of Brownian reorientation on fdp. Since spin relaxation and molecular reorienta- 


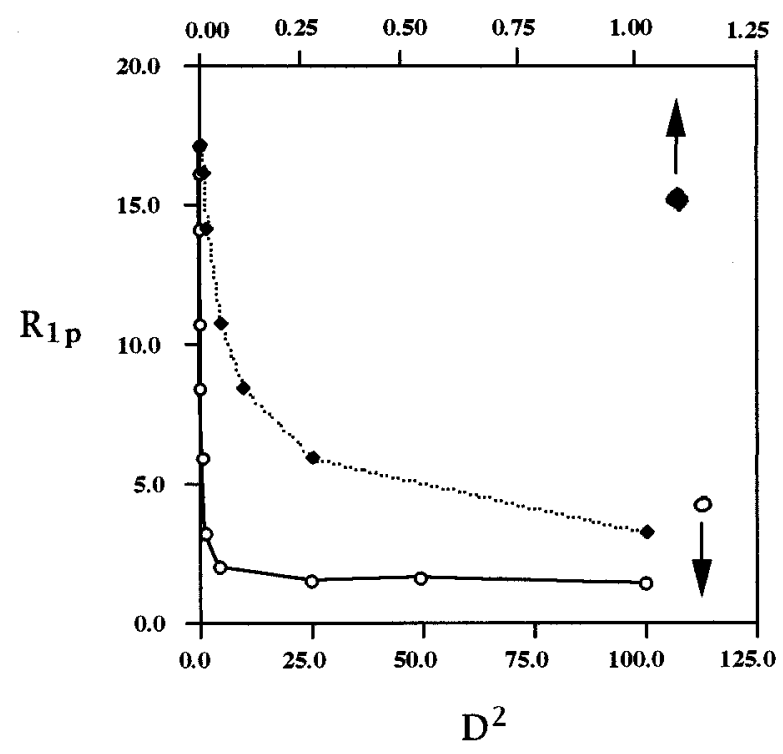

FIG. 9. Dependence of the NMR-PRE on $D$ (the uniaxial zfs parameter). Conditions are as in Fig. 5 except $\tau_{R}^{(1)}=3.0 \times 10^{-11} \mathrm{~s}$, and $D^{2}$ was permitted to vary. The values of $D^{2}$ for the filled diamonds range from 0.00 to 1.25 , top axis. The bottom axis describes the solid curve with open circles.

tion both act to randomize the dipolar interaction, shortening $\tau_{S, v}$ to the point where $\tau_{S, v} \leqslant \tau_{R}^{(1)}$ results in a decrease in $R_{1 p}$, and also tends to mask the effects of Brownian reorientation. This effect is shown in Fig. 10, where $R_{1 p}$ is plotted against $\tau_{R}^{(1)}$ for three values of $\tau_{S, v}: 0.1 \mathrm{~s}$ (open circles), $\tau_{S, v}=10^{-10} \mathrm{~s}$ (filled circles), and $\tau_{S, v}=10^{-11} \mathrm{~s}$ (diamonds). As expected, shorter $\tau_{S, v}$ acts to decrease $R_{1 p}$ and mask the effects of molecular reorientation.

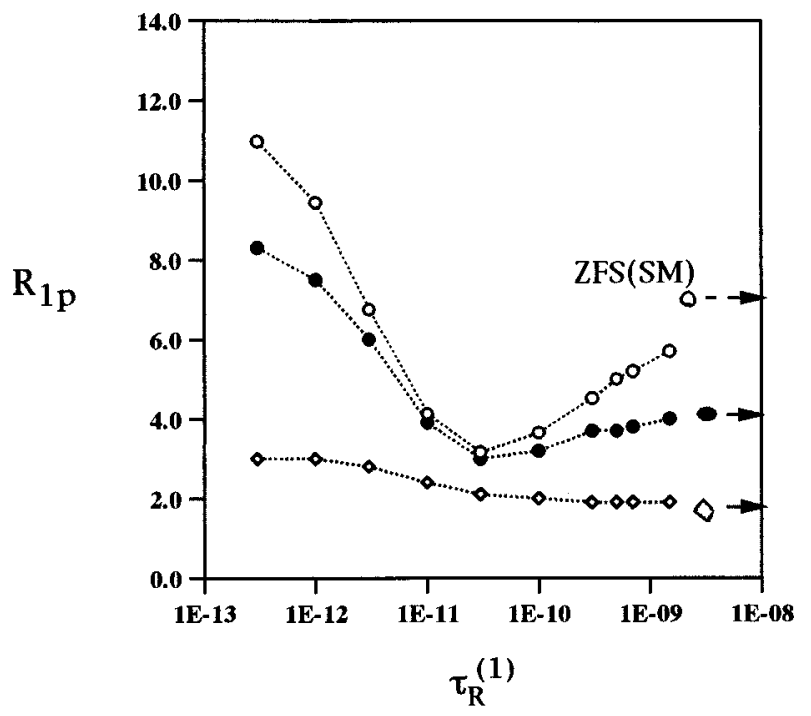

FIG. 10. $R_{1 p}$ as a function of reorientational correlation time $\tau_{R}^{(1)}$ for $S$ $=1$. Comparison of results obtained from SD simulations and intermolecular zfs-limit analytical theory. The slow motion zfs-limit [ZFS(SM)] result is shown as the short dashed line with an arrow. Other condition are given in the legend of Fig. 5, except for $\tau_{S, v}^{(0)}$ values, which were (a) $0.1 \mathrm{~s}$ (open circles), $1.0 \times 10^{-10} \mathrm{~s}$ (filled circles), and $1.0 \times 10^{-11} \mathrm{~s}$ (open diamonds).
It should be noted that all of the simulations described above assumed that $\tau_{v} \rightarrow 0$, i.e., that $\tau_{S, v}$ is independent of magnetic field strength. This approximation will break down at magnetic field strengths high enough that $2 \omega \tau_{v} \geqslant 1$, above which $\tau_{S, v}$ tends to a $B_{0}^{2}$ dependence. Since effects of molecular reorientation become important only when $\tau_{S}$ $<\tau_{R}^{(2)}$, field dependence in $\tau_{S}$ tends to increase the influence of reorientation on NMR-PRE phenomena.

\section{BROWNIAN REORIENTATION IN THE ANALYTICAL UNIAXIAL ZFS-LIMIT THEORY}

The analytical zfs-limit theory of Ref. 5 assumes a uniaxial zfs tensor $(E=0)$. The resulting analytical expressions are simple in form and mirror the well-known expression of the Zeeman-limit SBM theory. The assumptions underlying the analytical zfs-limit theory ${ }^{5}$ are the following: (1) that the zfs interaction is large $\left(H_{\mathrm{zfs}} \gg H_{\text {Zeem }}\right)$; (2) uniaxial $(E=0)$; and (3) that Brownian reorientation is slow enough to produce zfs-type precession (i.e., $\tau_{R}^{(2)} \omega_{D}>1$ ). The theory is not otherwise a "slow motion" theory, since the effects of Brownian reorientation in randomizing the dipolar interaction enter through the definition of the dipole-dipole correlation time, $\tau_{d d}$,

$$
\tau_{d d}^{-1}=\tau_{S, v}^{-1}+\tau_{S, r}^{-1}+\left(\tau_{R}^{(1)}\right)^{-1},
$$

where $\tau_{R}^{(1)}$, rather than $\tau_{R}^{(2)}$, appears for reasons that are described in Ref. 11. Figure 5 shows that this analytical theory provides an accurate description of the effects of Brownian reorientation throughout the regime of zfs-type precession, and that the theory becomes invalid when $\tau_{R}^{(2)} \omega_{D}<1$ as expected.

\section{COMPARISON WITH EXPERIMENTAL DATA FOR [Mn(III) (acac) $)_{3}$ ]}

[tris-(acetylacetonato) $\mathrm{Mn}(\mathrm{III})]\left(\mathrm{Mn}^{\mathrm{III}}(\mathrm{acac})_{3}\right)$ is a model $S=2$ spin system for which extensive intermolecular ${ }^{1} H$ NMR relaxation data have been reported at magnetic field strengths corresponding to the zfs limit and to the intermediate $\left(H_{\text {Zeem }} \approx H_{\text {zfs }}\right)$ regimes. An analysis of solvent (acetone) ${ }^{1} H$ NMR-PRE data has previously been carried out using the analytical zfs-limit theory of Ref. 5, in which the contribution of $\tau_{R}^{(1)}$ in Eq. (25) was included, but that of $\tau_{S, r}^{-1}$ was neglected. Effects of zfs rhombicity were considered in a subsequent study, ${ }^{13}$ using modified zfs-limit theory appropriate to the slow reorientation limit. Using the SD methods described above, we are now able to assess quantitatively the effects of molecular reorientation on the analysis.

Figure 11 shows the effect of varying the $\tau_{R}^{(1)}$ on $R_{1 p}$ values computed using physical parameters appropriate for $\mathrm{Mn}^{\mathrm{III}}(\mathrm{acac})_{3}$. Experimental $R_{1 p}$ data collected at field strengths of $0.3 \mathrm{~T}$ and $0.5 \mathrm{~T}$ and at a temperature of $298 \mathrm{~K}$ are also shown. The Debye value for $\tau_{R}^{(1)}$ for $\mathrm{Mn}^{\mathrm{III}}(\mathrm{acac})_{3}$ is approximately $120 \mathrm{ps}$, indicated by an arrow in the figure. The open symbols in Fig. 11 show the results of simulations in which $\tau_{R}^{(1)}$ was allowed to vary from the slow motion limit to $\tau_{R}^{(1)}=1 \times 10^{-11} \mathrm{~s}$. In these simulations, which were performed using the value $\tau_{S, v}=10 \mathrm{ps}$ as inferred in Ref. 5, the 


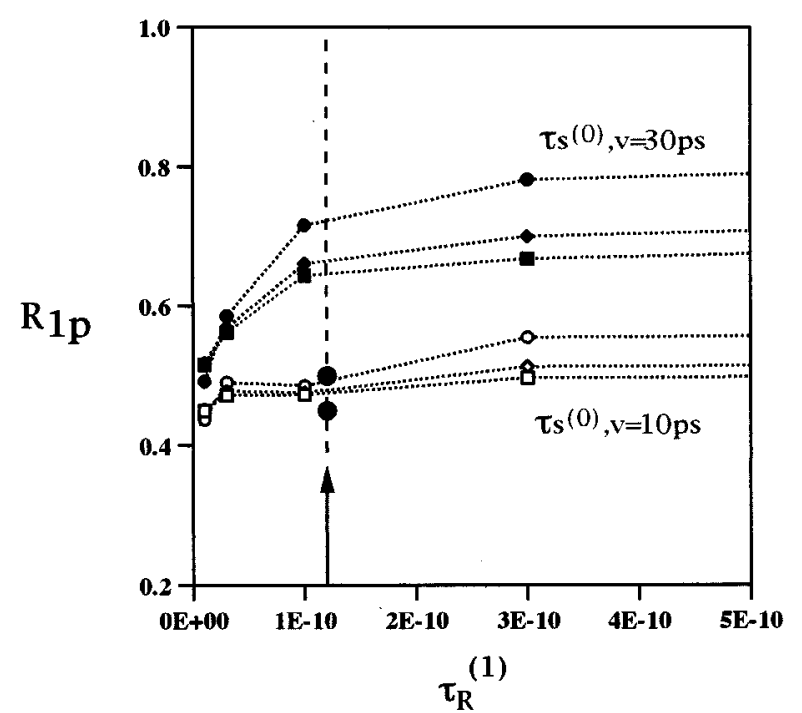

FIG. 11. $R_{1 p}$ as a function of reorientational correlation time for a $S=2$ spin system at $\tau_{S, v}^{(0)}=3 \times 10^{-11} \mathrm{~s}$ (filled symbols) and $1.0 \times 10^{-11} \mathrm{~s}$ (open symbols), with $\tau_{v} \rightarrow 0$, computed by spin dynamics (SD) simulation. The computations were done assuming $B_{0}=0.3 T$ (squares), $0.5 T$ (diamonds), and $1.0 T$ (circles), and assumed zfs parameters of $D=3.1 \mathrm{~cm}^{-1}$ (Ref. 47) and $E / D=0.05$. The molecular self diffusion coefficients of the solvent and solute were $D_{1}=4.5 \times 10^{-9}$ and $D_{2}=1.6 \times 10^{-9} \mathrm{~m}^{2} \mathrm{~s}^{-1}$, respectively, and the distance of closest approach was $d_{c}=0.43 \mathrm{~nm}$. The two large circles correspond to experimental data at 0.3 and $0.5 \mathrm{~T}$. The arrow denotes the Debye value for $\tau_{R}^{(1)}$ for $\mathrm{Mn}(\mathrm{acac})_{3}$ in acetone at $298 \mathrm{~K}$.

system is well represented by the slow motion approximation: the deviation between the slow motion analytical theory and the spin dynamics simulation when $\tau_{R}^{(1)}=120 \mathrm{ps}$ is $<10 \%$.

The result that Brownian reorientation is unimportant in the analysis of these data stems from the shortness of $\tau_{S, v}$ : we expect in general that molecular reorientation will have little effect on $R_{1 p}$ when $\tau_{R}^{(1)} \gg \tau_{S, v}$, since then spin relaxation tends to mask the effects of Brownian reorientation. This behavior was confirmed in the simulations. To test this point further, simulations were performed using the same physical parameters as described above, except that $\tau_{S, v}$ was lengthened to $30 \mathrm{ps}$ (solid symbols in Fig. 11). As $\tau_{S, v}$ lengthened, the simulation became increasingly sensitive to effects of rapid reorientational motion as expected, although at $\tau_{R}^{(1)}=120 \mathrm{ps}$ the deviation from the slow motion limit with $\tau_{S, v}=30 \mathrm{ps}$ is still quite small, $<10 \%$.

\section{SUMMARY}

Spin dynamics simulations as implemented in the program SpinDyn.f have been shown to provide accurate, flexible, and reasonably rapid calculations of NMR-PRE phenomena with few of the assumptions or limitations of previous analytical theories. The program calculates interand intramolecular NMR-PRE phenomena for both integer and half-integer spins precessing under arbitrary Zeeman and zfs interactions the presence of Brownian reorientation. The reorientational part of the electron spin relaxation rate $\tau_{S, r}^{-1}$ is not parametrized but is calculated directly from the static zfs parameters $D$ and $E$ by a non-Redfield approach. Two significant assumptions of the program in its present form are: first, that solute molecules reorient as rigid, isotropic units; and second, its use of the Redfield expression for $\tau_{S, v}$ [Eqs. (22)-(24)], thus introducing the parameters $\tau_{S, v}^{(0)}$ and $\tau_{v}$ into the simulation. The program can readily be modified to accommodate more complex reorientational models or, alternatively, to operate in conjunction with a molecular dynamics simulation of the molecular degrees of freedom. A priori quantum mechanical calculation of $\tau_{S, v}^{(0)}$ is also possible, ${ }^{29,30}$ although at present this kind of calculation is difficult and of limited accuracy. In most practical analyses, a parametric description of $\tau_{S, v}$ as we have used here seems useful.

An objective of this study was a better understanding of the influence of Brownian reorientation on NMR-PRE phenomena. It has been shown that the intermolecular uniaxial zfs-limit analytical theory ${ }^{5}$ describes the effects of molecular reorientation adequately when a dipolar correlation time of the functional form of Eqs. (25) and (26) is employed, and when electron spin precession has zfs character $\left(\omega_{D} \tau_{R}^{(2)}\right.$ $\gg 1$ ). Reorientational deviations from the analytical theories are large when Brownian reorientation is sufficiently rapid that $\omega_{D} \tau_{R}^{(2)} \leqslant 1$, in which case spin precession has Zeeman, rather than zfs, character. For the "slow motion" theories of the intermediate regime, ${ }^{6-11,14}$ as well as for the uniaxial zfs-limit expression, ${ }^{4,5}$ the effects of the Brownian reorientation on $R_{1 p}$ may be neglected when electron spin relaxation is rapid on the reorientational time scale $\left[\tau_{S, v} \ll \tau_{R}^{(2)}\right.$, see Eq. (25)], a point illustrated by the simulations of Fig. 10.

Summarizing the conditions for validity of the simple uniaxial zfs-limit analytical expressions of Refs. 4 and 5, the assumptions are (i) that $H_{\mathrm{zfs}} \gg H_{\text {Zeem }}$, (ii) that the zfs tensor be quadratic and uniaxial $(E \approx 0)$, and (iii) that electron spin precession have zfs character $\left(\omega_{D} \tau_{R}^{(2)} \gg 1\right)$. When these conditions are met, the effects of reorientation can be accounted for using Eqs. (25) and (26). The conditions for validity of the Zeeman-limit analytical theory are simpler than those for zfs-limit theories, namely, that $H_{\text {Zeem }} \gg H_{\text {zfs }}$ or that $\omega_{D} \tau_{R}^{(2)}$ $\ll 1$ (either condition suffices).

\section{ACKNOWLEDGMENTS}

This research was supported by the U. S. National Science Foundation in the form of a research grant, CHE9423351. S.M.A. received financial support in the form of a Rackham Merit Fellowship from the University of Michigan.

${ }^{1}$ I. Solomon, Phys. Rev. 99, 559 (1955).

${ }^{2}$ N. Bloembergen, J. Chem. Phys. 27, 572, 595 (1957).

${ }^{3}$ N. Bloembergen and L. O. Morgan, J. Chem. Phys. 34, 842 (1961).

${ }^{4}$ R. R. Sharp, J. Chem. Phys. 93, 6921 (1990).

${ }^{5}$ T. Bayburt and R. R. Sharp, J. Chem. Phys. 92, 5892 (1990).

${ }^{6}$ I. Bertini, C. Luchinat, M. Mancini, and G. Spina, J. Magn. Reson. 59, 213 (1984).

${ }^{7}$ I. Bertini, C. Luchinat, and J. Kowalewski, J. Magn. Reson. 62, 235 (1985).

${ }^{8}$ L. Banci, I. Bertini, F. Briganti, and C. Luchinat, J. Magn. Reson. 66, 58 (1986).

${ }^{9}$ H. Fukui, K. Miura, and H. Matsuda, J. Magn. Reson. 88, 311 (1990).

${ }^{10}$ R. R. Sharp, J. Magn. Reson. 100, 491 (1992).

${ }^{11}$ R. R. Sharp, J. Chem. Phys. 98, 912 (1993). 
${ }^{12}$ R. R. Sharp, J. Chem. Phys. 98, 2507 (1993).

${ }^{13}$ J.-M. Bovet and R. R. Sharp, J. Chem. Phys. 99, 18 (1993).

${ }^{14}$ I. Bertini, O. Galas, C. Luchinat, and G. Parigi, J. Magn. Reson. A 113, 151 (1995).

${ }^{15}$ J. H. Freed, G. V. Bruno, and C. Polnaszek, J. Chem. Phys. 55, 5270 (1971).

${ }^{16}$ J. H. Freed, G. V. Bruno, and C. Polnaszek, J. Chem. Phys. 55, 716 (1972).

17 T.-H. R. Chen, S.-J. Den, and L.-P. Hwang, Proc. Nat. Sci. Council (Taiwan) 8, 224 (1984).

${ }^{18}$ L.-P. Hwang and C.-Y. Ju, J. Chem. Phys. 83, 3775 (1985),

${ }^{19}$ P.-L. Wang, J.-H. Lee, S.-M. Huang, and L.-P. Hwang, J Magn. Reson. 73, 277 (1987).

${ }^{20}$ N. Benetis, J. Kowalewski, L. Nordenskiold, H. Wennerstrom, and P.-O. Westlund, Mol. Phys. 48, 329 (1983).

${ }^{21}$ N. Benetis, J. Kowalewski, L. Nordenskiold, H. Wennerstrom, and P.-O. Westlund, J. Magn. Reson. 58, 261 (1984).

${ }^{22}$ P.-O. Westlund, H. Wennerstrom, L. Nordenskiold, J. Kowalewski, and N. Benetis, J. Magn. Reson. 59, 91 (1984).

${ }^{23}$ N. Benetis and J. Kowalewski, J Magn. Reson. 65, 13 (1985).

${ }^{24}$ T. Larsson, P.-O. Westlund, J. Kowalewski, and S. H. Koenig, J. Chem. Phys. 101, 1116 (1994).

${ }^{25}$ N. Benetis, J. Kowalewski, L. Nordenskiold, and U. Edlund, J. Magn. Reson. 58, 282 (1984).

${ }^{26}$ P.-O. Westlund, in Dynamics of Solutions and Fluid Mixtures by NMR, edited by J. J. Delpuech (Wiley and Sons, New York, 1995), p. 173.

${ }^{27}$ P. A. Eglestaff, J. Chem. Phys. 53, 2590 (1970).

${ }^{28}$ S. Alexander, A. Baram, and Z. Luz, Mol. Phys. 27, 441 (1974).
${ }^{29}$ M. Odelius, C. Ribbing, and J. Kowalewski, J. Chem. Phys. 103, 1800 (1995)

${ }^{30}$ M. Odelius, C. Ribbing, and J. Kowalewski, J. Chem. Phys. 104, 3181 (1996).

${ }^{31}$ T. Bayburt and R. R. Sharp, J. Phys. Chem. 97, 4558 (1993).

${ }^{32}$ H. C. Torrey, Phys. Rev. 92, 962 (1953).

${ }^{33}$ H. Pfeifer, Ann. Phys. Leipzig 7, 1 (1961).

${ }^{34}$ A. Abragam, The Principles of Nuclear Magnetism (Oxford University, Oxford, 1961), pp. 300-302.

${ }^{35}$ L.-P. Hwang and J. H. Freed, J. Chem. Phys. 63, 4017 (1975).

${ }^{36}$ H. L. Friedman, M. Holz, and H. G. Hertz, J. Chem. Phys. 70, 3369 (1969)

${ }^{37}$ W. H. Press, S. A. Teukolsky, W. T. Vetterling, B. P. Flannery, Numerical Recipes in Fortran, 2nd ed. (Cambridge University Press, Cambridge, 1994).

${ }^{38}$ L. Banci, I. Bertini, and C. Luchinat, Nuclear and Electron Relaxation (VCH, New York, 1991).

${ }^{39}$ A. D. McLachlan, Proc. Roy. Soc. A 280, 27 (1964).

${ }^{40}$ A. Carrington and G. R. Luckhurst, Mol. Phys. 8, 125 (1964).

${ }^{41}$ A. Hudson and G. R. Luckhurst, Mol. Phys. 16, 395 (1969).

${ }^{42}$ A. Al'tshuler and K. A. Valiev, Sov. Phys. JETP 8, 661 (1959).

${ }^{43}$ N. Bloembergen and L. O. Morgan, J. Chem. Phys. 34, 842 (1961).

${ }^{44}$ M. Rubinstein, A. Baram, and Z. Luz, Mol. Phys. 20, 67 (1971).

${ }^{45}$ J. E. Roberts and J. Schnittker, J. Phys. Chem. 97, 5410 (1993).

${ }^{46}$ P.-O. Westlund, Mol. Phys. 85, 1165 (1995).

${ }^{47}$ A. K. Gregson, D. M. Doddrell, and P. C. Healy, Inorg. Chem. 17, 1216 (1978) 